\title{
Efficacy and Safety of Belatacept Treatment in Renal Allograft Recipients at High Cardiovascular Risk-A Single Center Experience
}

\author{
Hannes Neuwirt ${ }^{1, * \mathbb{D}}$, Irmgard Leitner-Lechner ${ }^{2}$, Julia Kerschbaum ${ }^{1}$, Michael Ertl ${ }^{1}$, \\ Florian Pöggsteiner ${ }^{1}$, Nicolas Pölt ${ }^{1}$, Julius Mätzler ${ }^{1}$, Hannelore Sprenger-Mähr ${ }^{3}$, \\ Michael Rudnicki ${ }^{1}$, Peter Schratzberger ${ }^{1}$, Iris E. Eder ${ }^{4}$ and Gert Mayer ${ }^{1}$ \\ 1 Department of Internal Medicine IV-Nephrology and Hypertension, Medical University of Innsbruck, \\ Anichstrasse 35, Innsbruck A-6020, Austria \\ 2 Department of Pharmacology, Section for Molecular and Cellular Pharmacology, Medical University of \\ Innsbruck, Peter-Mayr-Strasse 1a, Innsbruck 6020, Austria \\ 3 Landeskrankenhaus Feldkirch, Department of Internal Medicine III-Nephrology, Carinagasse 47, \\ Feldkirch 6800, Austria \\ 4 Department of Urology, Medical University of Innsbruck, Anichstrasse 35, Innsbruck A-6020, Austria \\ * Correspondence: Hannes.Neuwirt@i-med.ac.at
}

Received: 2 July 2019; Accepted: 1 August 2019; Published: 3 August 2019

\begin{abstract}
Belatacept is an attractive option for immunosuppression after renal transplantation. Renal allograft function is superior when compared to calcineurin inhibitor (CNI) based therapy in "de novo" treated patients and it has also been proposed that individuals at high cardiovascular (CV) risk may benefit most. In this retrospective cohort study, we assessed the efficacy and safety of treating patients at high cardiovascular risk with Belatacept ( $n=34$, for 1194 observation months) when compared to a matched control group of 150 individuals under CNI immunosuppression (for 7309 months of observation). The estimated glomerular filtration rate (eGFR) increased for patients taking Belatacept but decreased during CNI-based therapy (+2.60 vs. $-0.89 \mathrm{~mL} / \mathrm{min} / 1.73 \mathrm{~m}^{2} /$ year, $p=0.006$ ). In a multivariate Cox regression model, Belatacept remained the only significant factor associated with the improvement of eGFR (HR 4.35, 95\%CI 2.39-7.93). Belatacept treatment was not a significant risk factor for renal allograft rejection or graft loss. In terms of safety, the only significant risk factor for de novo cardiovascular events was a pre-existing cerebrovascular disease, but Belatacept was not associated with a significant risk reduction. Belatacept treatment was not associated with an increased risk of severe infections, cytomegalo virus (CMV) or BK-virus reactivation, malignancy or death in the multivariate Cox regression analysis. Belatacept is an efficient and safe option for patients after renal transplantation at high cardiovascular risk.
\end{abstract}

Keywords: kidney transplantation; Belatacept; cardiovascular high risk; outcome

\section{Introduction}

Calcineurin inhibitors (CNIs) are currently the standard immunosuppressive therapy after renal transplantation. Their introduction into clinical practice has improved short-term outcomes dramatically. Unfortunately, the rate of late allograft loss has not significantly improved [1] and it is generally accepted that CNI nephrotoxicity contributes to this problem. Thus, multiple studies have investigated the impact of CNI-free immunosuppression on renal allograft function and patient and graft survival. The use of mammalian target of rapamycin inhibitors (mTORi) is impeded by drop-out rates of up to $40 \%$ due to side effects [2] and furthermore is associated with higher allograft rejection rates [3]. 
Belatacept inhibits T-cell activation by blocking a co-stimulatory signal by binding to CD80/CD86 on antigen presenting cells [4]. It is currently approved for the de novo immunosuppression of renal allograft recipients in combination with mycophenolic acid and steroids. Studies have demonstrated an improved allograft survival over several years when compared to cyclosporine A-based immunosuppression [5,6]. Furthermore, a protocol for switching patients from CNIs to Belatacept has been published $[7,8]$. This conversion improved kidney function relative to the baseline and was safe concerning risk of death or transplant loss. Finally, it has been proposed that Belatacept-based regimens might have beneficial effects, especially in patients at high cardiovascular (CV) risk (reviewed by [9]). One mechanism might be a reduction of pulse wave velocity in patients treated with Belatacept compared to CNI-treated patients $[10,11]$. However, "real world" data on renal outcomes and especially safety in the latter individuals are sparse.

Thus, we conducted a retrospective cohort study in renal allograft recipients at high CV risk and compared the efficacy and safety of Belatacept treatment in 34 patients to the outcomes of 150 patients treated with CNI (mainly tacrolimus) based immunosuppression.

\section{Materials and Methods}

\subsection{Patient Population}

Belatacept has been used in 42 renal transplant recipients at our center since 2010 and in this retrospective cohort study, all patients at high cardiovascular risk (definition see below) have been included $(n=34)$. Eighteen patients were treated de novo and 16 were converted at a median of 1.6 months (interquartile range (IQR), 0.6-4.2 months) after transplantation, mainly due to biopsy confirmed or clinically suspected renal CNI toxicity. No patient in this group was returned to CNI therapy thereafter. As we were interested in studying the efficacy and safety in patients on either CNI or Belatacept therapy, the day of conversion was taken as the baseline in these individuals and all clinical endpoints were adjudicated to the Belatacept group. Due to the early conversion, we excluded the time on CNI from any calculation. The study was conducted in accordance with the Declaration of Helsinki, and the protocol was approved by the Ethics Committee of the Medical University Innsbruck (Project-ID: 1137/2019).

The median observation duration on Belatacept was 35 months, no patient was lost for follow-up and the total period on therapy analysed was 1194 months. All renal allograft recipients $(n=309)$ on CNI-based immunosuppression that received their transplant between 1 January, 2010 and 31 December, 2012 formed the control cohort. Of these, 150 also fulfilled the criteria for high cardiovascular risk. No patients were lost for follow-up, the median follow-up was 48 months and the total analysed period of months on therapy was 7309. High cardiovascular risk was defined by the presence of any significant pre-transplant coronary artery disease (CAD) confirmed on angiography, a history of myocardial infarction, peripheral artery disease (PAD) (cardiovascular disease) or stroke (cerebrovascular disease) or the presence of diabetes mellitus in combination with arterial hypertension.

\subsection{Endpoints}

Efficacy endpoints were renal allograft function as assessed by a change of eGFR on therapy, number of rejection episodes (either confirmed by biopsy or clinically based on an improvement of allograft function after anti-rejection therapy) or graft loss. The estimated glomerular filtration rate (eGFR) was calculated using the abbreviated MDRD formula. $\triangle$ eGFR was calculated by dividing the difference between eGFR at last follow-up and the baseline by the number of follow-up years. The safety endpoints were de novo cardiovascular events (new myocardial infarction, newly diagnosed CAD of any stage, newly diagnosed peripheral artery disease), severe infections (defined as infection leading to the admission of the patient to hospital), cytomegalo virus (CMV) reactivation (diagnosed by PCR with or without a clinical CMV infection), BKV reactivation (as determined by PCR in serum 
and/or urine), de novo malignancy and death. All efficacy and safety endpoints were identified using patients' records.

\subsection{Statistics}

The reported values represent either medians and interquartile ranges (IQR) or the number of patients and percentages of the respective cohort. Proportions were compared using the $\mathrm{Chi}^{2}$ or Fisher exact tests. Non-parametric tests were used to compare continuous variables. The factors potentially associated with the eGFR, the eGFR-slope (eGFR), and efficacy and safety parameters were assessed using a Cox regression analysis. In particular, those factors were: Belatacept treatment, recipient age, male gender of the recipient, recipient BMI, a CMV high risk mismatch (D+/R-), the presence of diabetes mellitus or arterial hypertension, the presence of cerebrovascular or cardiovascular disease, the time on renal replacement therapy (RRT) before renal transplantation (RTx), number of previous RTx, number of HLA mismatches, intraoperative urine production (initial diuresis), number of post-operative (PO) - meaning after renal transplantation-hemodialysis sessions (HDs), the absence or presence of steroids at discharge, the presence of serum-creatinine at discharge, the absence or presence of steroids at the last follow-up, the extended criteria donor (ECD) organ, the male sex of the donor, and donor age. A history of rejection was also included, with an exception for the endpoint rejection episodes. Variables with a $p$-value $<0.05$ in univariate analysis were included in the multivariate analysis, where again a $p$-value $<0.05$ was considered statistically significant. The analysis was performed using SPSS Version 24.

\section{Results}

\subsection{Baseline}

Baseline data are shown in Table 1. Belatacept patients spent a shorter period of time on renal replacement therapy (RRT) before renal transplantation, were more likely to suffer from CV disease or hypertension and less likely to have diabetes mellitus compared to CNI patients. The baseline data were not significantly different between de novo and converted Belatacept patients, except for the higher proportion of male recipients in de novo patients $(17 / 18$ vs. $8 / 16, p=0.003)$.

\subsection{Renal Transplantation}

Concerning renal transplantation (RTx, Table 2), the donor type was significantly different between CNI- and Belatacept-treated patients. This was primarily driven by a higher proportion of living donors and deceased donors that died due to circulatory reasons in the Belatacept group. The Belatacept patients received more organs from female donors and donors were older (61 vs 49.5 years) and had a higher BMI compared to CNI patients. The proportion of patients with intraoperative urine production (initial diuresis) was lower (76\% vs. $95 \%$ ) and the number of hemodialysis sessions (HDs) was significantly higher in Belatacept patients. Hence, renal allograft function at discharge, as assessed by serum creatinine ( 1.52 vs. $2.20 \mathrm{mg} / \mathrm{ml}, p=0.001)$ and eGFR (MDRD) $\left(44.5 \mathrm{vs.} 28 \mathrm{~mL} / \mathrm{min} / 1.73 \mathrm{~m}^{2}\right.$, $p=0.001)$, was significantly worse in Belatacept patients.

\subsection{Efficacy}

The median follow-up (Table 3) was 1462 and 1054 days in CNI and Belatacept patients $(p=0.084)$, respectively. Belatacept was continued in all patients with a maintained graft function during follow-up (31/34). The number of patients on steroids at follow-up and the proportion of hypertensive patients were higher in the Belatacept group. 
Table 1. Baseline data of control and Belatacept patients.

\begin{tabular}{|c|c|c|c|c|c|}
\hline & \multicolumn{2}{|c|}{ Control $(n=150)$} & \multicolumn{3}{|c|}{ Belatacept $(n=34)$} \\
\hline & Median $/ n$ & IQR/(\%) & Median $/ n$ & IQR/(\%) & $p$ \\
\hline BMI $\left[\mathrm{kg} / \mathrm{m}^{2}\right]$ & 25.1 & $22.6-28.4$ & 26.1 & $22.6-30.6$ & 0.304 \\
\hline Male Sex $n(\%)$ & 37 & $(24.67)$ & 9 & $(26.47)$ & 0.826 \\
\hline Age at time of RTx [years] & 59.6 & $49.4-66.8$ & 57.2 & $38.7-65.4$ & 0.325 \\
\hline Time on RRT [months] & 53.8 & $29.6-80.5$ & 35.6 & $22.2-52.1$ & $0.006 *$ \\
\hline \multicolumn{6}{|l|}{ Primary Renal Disease $n(\%)$} \\
\hline Diabetic Nephropathy & 51 & $(34.00)$ & 5 & (14.71) & $0.031 *$ \\
\hline Vascular Nephropathy & 25 & $(16.67)$ & 5 & $(14.71)$ & 0.768 \\
\hline IgA Nephropathy & 7 & $(4.67)$ & 6 & $(17.65)$ & $0.017 *$ \\
\hline other Glomerulonephritis & 18 & (12.00) & 2 & $(5.88)$ & 0.377 \\
\hline ADPKD & 12 & $(8.00)$ & 5 & (14.71) & 0.321 \\
\hline other hereditary disease & 2 & (1.33) & 0 & $(0.00)$ & 1.000 \\
\hline ANCA Vasculitis & 0 & $(0.00)$ & 0 & $(0.00)$ & n.c. \\
\hline Lupus nephropathy & 2 & (1.33) & 0 & $(0.00)$ & 1.000 \\
\hline chronic kidney disease NOS & 33 & (22.00) & 11 & (32.35) & 0.209 \\
\hline Number of previous RTx $n(\%)$ & & & & & 0.671 \\
\hline 0 & 114 & (76.00) & 29 & (85.29) & \\
\hline 1 & 24 & (16.00) & 4 & (11.76) & \\
\hline 2 & 10 & $(6.67)$ & 1 & $(2.94)$ & \\
\hline 3 & 1 & $(0.67)$ & 0 & $(0.00)$ & \\
\hline 4 & 1 & $(0.67)$ & 0 & $(0.00)$ & \\
\hline Diabetes $n(\%)$ & 85 & (56.67) & 11 & $(32.35)$ & 0.009 * \\
\hline Cerebrovascular disease $n(\%)$ & 12 & $(8.00)$ & 4 & (11.76) & 0.503 \\
\hline Cardiovascular disease $n(\%)$ & 113 & (75.33) & 32 & (94.12) & 0.016 * \\
\hline Arterial Hypertension $n(\%)$ & 126 & $(84.00)$ & 34 & $(100)$ & 0.014 * \\
\hline
\end{tabular}

The median and interquartile range (IQR) are depicted except for nominal variables, where the number of patients $(n)$ and percentages are shown. $p$-values $<0.05$ are marked with an asterisk *. BMI: body mass index, RTx: renal transplantation, RRT: renal replacement therapy, ADPKD: autosomal dominant polycystic kidney disease, ANCA: antineutrophil cytoplasmic antibody, NOS: not otherwise specified, n.c.: not calculated.

Table 2. Data at the time of Renal Transplantation (RTx).

\begin{tabular}{|c|c|c|c|c|c|}
\hline & \multicolumn{2}{|c|}{ Control $(n=150)$} & \multicolumn{3}{|c|}{ Belatacept $(n=34)$} \\
\hline & Median/n & IQR/(\%) & Median/n & IQR/(\%) & $p$ \\
\hline \multicolumn{6}{|l|}{ Donor Type $n(\%)$} \\
\hline Living donor & 6 & $(4.00)$ & 5 & (14.71) & $0.032 *$ \\
\hline DD (CVA/SAB/SDH) & 86 & (57.33) & 22 & $(64.71)$ & 0.431 \\
\hline DD (trauma) & 41 & (27.33) & 0 & $(0.00)$ & $0.001 *$ \\
\hline DD (circulatory) & 6 & $(4.00)$ & 5 & (14.71) & $0.032 *$ \\
\hline DD (suicide) & 7 & $(4.67)$ & 2 & $(5.88)$ & 0.673 \\
\hline $\mathrm{DD}$ (other) & 4 & $(2.67)$ & 0 & $(0.00)$ & 1.000 \\
\hline $\operatorname{ECD} n(\%)$ & 65 & (43.33) & 18 & (52.94) & 0.309 \\
\hline Male Donor Sex $n(\%)$ & 80 & $(53.33)$ & 9 & (26.47) & $0.005 *$ \\
\hline Donor Age [years] & 49.5 & $37-66$ & 61 & $51-68$ & $0.003 *$ \\
\hline Donor BMI $\left[\mathrm{kg} / \mathrm{m}^{2}\right]$ & 24.9 & $22.5-27.7$ & 26.3 & $24.5-28.6$ & 0.034 * \\
\hline CMV mismatch $n(\%)$ & & & & & 0.947 \\
\hline Donor-/Recipient- & 20 & (13.33) & 5 & $(14.71)$ & \\
\hline $\begin{array}{c}\text { Donor-/Recipient+ or } \\
\text { Donor+/Recipient+ }\end{array}$ & 100 & $(66.66)$ & 22 & $(64.71)$ & \\
\hline Donor+/Recipient- & 30 & $(20.00)$ & 7 & $(20.59)$ & \\
\hline Number of HLA Mismatches & 3 & $3-5$ & 3 & $2-5$ & 0.843 \\
\hline Initial Diuresis $n(\%)$ & 143 & (95.33) & 26 & $(76.47)$ & $<0.001 *$ \\
\hline Number of PO HDs after RTx & 0 & $0-2$ & 1 & $0-5$ & $0.015 *$ \\
\hline On Steroids at discharge $n(\%)$ & 141 & $(94.00)$ & 34 & $(100.00)$ & 0.214 \\
\hline S-Creatinine at discharge $(\mathrm{mg} / \mathrm{dL})$ & 1.52 & $1.21-2.11$ & 2.20 & $1.40-2.98$ & $0.001 *$ \\
\hline $\begin{array}{c}\text { eGFR at discharge (MDRD) } \\
\left(\mathrm{mL} / \mathrm{min} / 1.73 \mathrm{~m}^{2}\right)\end{array}$ & 44.5 & $30-9$ & 28 & $22-51$ & $0.001 *$ \\
\hline
\end{tabular}

Median and IQR are depicted except for nominal variables, where the number of patients $(n)$ and percentages are shown. $p$-values $<0.05$ are marked with an asterisk * DD: deceased donor, CVA: cerebrovascular event, SAB: subarachnoidal bleeding, SDH: subdural hematoma, ECD: extended criteria donor, CMV mismatch: "-" means sero-negative, "+" means sero-positive, PO HDs: postoperative hemodialysis sessions, RTx: renal transplantation, S-Creatinine: serum creatinine. 
Table 3. Data at last follow-up.

\begin{tabular}{|c|c|c|c|c|c|}
\hline & \multicolumn{2}{|c|}{ Control $(n=150)$} & \multicolumn{3}{|c|}{ Belatacept $(n=34)$} \\
\hline & Median $/ n$ & IQR/(\%) & Median/n & IQR/(\%) & $p$ \\
\hline Follow-up [days] & 1462 & $718-2115$ & 1054 & $772-1363$ & 0.084 \\
\hline IS-CNI/mTor/Bela $n(\%)$ & & & & & $<0.001 *$ \\
\hline none & 14 & 9.33 & 3 & 8.82 & \\
\hline $\mathrm{Tac}$ & 105 & 70.00 & 0 & 0.00 & \\
\hline CsA & 25 & 16.67 & 0 & 0.00 & \\
\hline Bela & 0 & 0.00 & 31 & 91.17 & \\
\hline mTORi & 6 & 4.00 & 0 & 0 & \\
\hline IS-Antimetabolites $n(\%)$ & & & & & 0.221 \\
\hline none & 32 & 21.33 & 5 & 14.71 & \\
\hline Mycophenolate Mofetil (MMF) & 75 & 50.00 & 19 & 55.88 & \\
\hline Mycophenolic acid (MPA) & 16 & 10.67 & 7 & 20.59 & \\
\hline Azathioprine & 25 & 16.67 & 2 & 5.88 & \\
\hline other & 2 & 1.33 & 1 & 2.94 & \\
\hline IS-On steroids $n(\%)$ & 71 & 47.33 & 28 & 82.35 & $<0.001 *$ \\
\hline Serum Creatinine (mg/dL) & 1.62 & $1.25-2.36$ & 1.96 & 1.342 .31 & 0.404 \\
\hline eGFR (MDRD) $\left(\mathrm{mL} / \mathrm{min} / 1.73 \mathrm{~m}^{2}\right)$ & 41 & $27-60$ & 36.5 & $25-52$ & 0.329 \\
\hline $\begin{array}{c}\triangle \mathrm{eGFR}(\mathrm{MDRD}) \\
\left(\mathrm{mL} / \mathrm{min} / 1.73 \mathrm{~m}^{2} / \text { year }\right)\end{array}$ & -0.89 & $-6.05-3.02$ & 2.6 & $-1.85-7.76$ & $0.006^{*}$ \\
\hline Cerebrovascular disease $n(\%)$ & 13 & 8.67 & 5 & 14.71 & 0.335 \\
\hline Cardiovascular disease $n(\%)$ & 118 & 78.67 & 33 & 97.06 & $0.004 *$ \\
\hline Arterial Hypertension $n(\%)$ & 120 & 80.00 & 30 & 88.24 & 1.000 \\
\hline
\end{tabular}

Concerning efficacy, renal allograft function as assessed by serum creatinine/eGFR improved in Belatacept-treated patients and slightly worsened in CNI patients, yielding a non-significant difference between the groups at follow-up (in contrast to the time of discharge after RTx). $\triangle \mathrm{eGFR}$ of the patients on Belatacept was positive compared to CNI patients (+2.60 vs. $-0.89 \mathrm{~mL} / \mathrm{min} / 1.73 \mathrm{~m}^{2} /$ year, $p=0.006$ ). The median $\triangle$ eGFR in the whole cohort (Belatacept plus CNI patients, $n=184$ ) was +0.35 $\mathrm{mL} / \mathrm{min} / 1.73 \mathrm{~m}^{2}$ (Table 4 ). The only factor significantly associated with a $\triangle \mathrm{eGFR}$ above the median in the multivariate model (adjusted for significant factors in the univariate analysis including Belatacept treatment, recipient BMI, number of postoperative HDs, the presence of serum-creatinine at discharge and donors' age) was Belatacept treatment (HR 4.35, 95\%CI 2.387-7.926, $p<0.001$, Table 4). Rejection episodes and graft loss were not significantly different between Belatacept and CNI patients (Table 5). Neither Belatacept nor any other parameter was a significant risk factor for rejection in the univariate Cox regression. Univariate correlated risk factors for graft loss were a higher recipient BMI (HR 1.126, 95\%CI 1.038-1.222, $p=0.004$ ), number of postoperative HDs (HR 1.178, 95\%CI 1.041-1.333, $p=0.009$ ) and higher serum-creatinine at discharge (HR 1.598, 95\%CI 1.179-2.166, $p=0.03$ ). BMI (HR 1.116, 95\%CI $1.003-1.242, p=0.043$ ) and number of postoperative HDs (HR 1.253, 95\%CI 1.027-1.530, $p=0.027$ ) remained significant after multivariate adjustments. Belatacept was not a risk factor for graft loss (HR $0.987,95 \%$ CI $0.283-3.417, p=0.980$ ). 
Table 4. Cox regression for eGFR better than the median $\left(+0.35 \mathrm{~mL} / \mathrm{min} / 1.73 \mathrm{~m}^{2} /\right.$ year $)$ at last follow-up. We calculated univariate hazard ratios (HR) for risk factors. All significant univariate risk factors were used in the multivariate model. BMI: body mass index, RTx: renal transplantation, PO HD: postoperative hemodialysis session, ECD: extended criteria donor.

\begin{tabular}{|c|c|c|c|c|c|c|}
\hline \multirow{2}{*}{ Factor } & \multicolumn{3}{|c|}{ Univariate Analysis } & \multicolumn{3}{|c|}{ Multivariate Analysis ${ }^{1}$} \\
\hline & HR & $95 \%-C I$ & $p$ & HR & $95 \%-C I$ & $p$ \\
\hline Belatacept & 5.629 & $3.239-9.783$ & $<0.001 *$ & 4.350 & $2.387-7.926$ & $<0.001$ * \\
\hline Recipient Age & 1.012 & $0.994-1.030$ & 0.184 & & & \\
\hline Male recipient Sex & 1.435 & $0.863-2.388$ & 0.164 & & & \\
\hline BMI & 1.063 & $1.018-1.111$ & $0.006^{*}$ & 1.024 & $0.979-1.072$ & 0.300 \\
\hline Diabetes & 0.799 & $0.524-1.218$ & 0.297 & & & \\
\hline Cerebrovascular disease & 1.365 & $0.704-2.646$ & 0.357 & & & \\
\hline Cardiovascular disease & 1.050 & $0.624-1.767$ & 0.853 & & & \\
\hline Arterial hypertension & 0.846 & $0.475-1.508$ & 0.572 & & & \\
\hline Number of previous RTx & 0.741 & $0.499-1.098$ & 0.135 & & & \\
\hline HLA mismatch & 0.649 & $0.416-1.011$ & 0.056 & & & \\
\hline Initial Diuresis & 0.502 & $0.200-1.260$ & 0.142 & & & \\
\hline Number of PO HDs & 1.117 & $1.051-1.187$ & $<0.001 *$ & 1.037 & $0.953-1.129$ & 0.395 \\
\hline Steroid at discharge & 2.424 & $0.760-7.731$ & 0.134 & & & \\
\hline Creatinine at discharge & 1.437 & $1.238-1.667$ & $<0.001$ * & 1.218 & $0.885-1.675$ & 0.226 \\
\hline ECD & 1.357 & $0.891-2.066$ & 0.154 & & & \\
\hline Male Donor Sex & 1.131 & $0.742-1.725$ & 0.566 & & & \\
\hline Donor Age & 1.018 & $1.005-1.032$ & 0.007 * & 1.004 & 0.990-1.019 & 0.546 \\
\hline
\end{tabular}

${ }^{1}$ adjusted for Belatacept treatment, BMI, number of postoperative (PO) hemodialysis sessions (HDs), eGFR at discharge and donor age. $p$-values $<0.05$ are marked with an asterisk*.

Table 5. Efficacy and safety endpoints at last follow-up. $p$-values $<0.05$ are marked with an asterisk * CMV: cytomegalo virus, BKV: polyoma virus.

\begin{tabular}{|c|c|c|c|c|c|c|}
\hline \multirow[b]{2}{*}{ EFFICACY } & \multicolumn{2}{|c|}{ CNI $(n=150)$} & \multicolumn{4}{|c|}{ Belatacept $(n=34)$} \\
\hline & $n$ & $\%$ & $n$ & $\%$ & $p$ & Log-Rank \\
\hline Rejection & 13 & 8.7 & 4 & 11.8 & 0.524 & 0.295 \\
\hline Graft loss & 17 & 11.3 & 3 & 8.8 & 1.000 & 0.980 \\
\hline \multicolumn{7}{|l|}{ SAFETY } \\
\hline De novo CV events & 5 & 3.33 & 1 & 2.94 & 1.000 & 0.550 \\
\hline Severe Infection & 35 & 23.3 & 13 & 38.2 & 0.074 & $0.013^{*}$ \\
\hline Type of severe Infection & & & & & $0.003 *$ & \\
\hline none & 115 & 76.7 & 21 & 61.8 & & \\
\hline Diarrhea & 1 & 0.7 & 3 & 8.8 & & \\
\hline Urinary tract infection & 15 & 10.0 & 6 & 17.6 & & \\
\hline Pneumonia & 15 & 10.0 & 1 & 2.9 & & \\
\hline Sepsis & 4 & 2.7 & 3 & 8.8 & & \\
\hline Any CMV reactivation & 60 & 40.0 & 16 & 47.1 & 0.450 & 0.932 \\
\hline BKV reactivation in serum & 16 & 10.7 & 7 & 20.6 & 0.148 & 0.136 \\
\hline BKV reactivation in urine & 37 & 24.7 & 10 & 29.4 & 0.567 & 0.718 \\
\hline Malignancy & 2 & 1.3 & 0 & 0.0 & 1.000 & 0.650 \\
\hline Death & 22 & 14.7 & 4 & 11.8 & 0.790 & 0.861 \\
\hline Cause of Death & & & & & 0.921 & \\
\hline unknown & 5 & 3.3 & 1 & 2.9 & & \\
\hline Sepsis & 7 & 4.7 & 2 & 5.9 & & \\
\hline Cardiac & 7 & 4.7 & 1 & 2.9 & & \\
\hline Malignancy & 2 & 1.3 & 0 & 0.0 & & \\
\hline Stroke & 1 & 0.7 & 0 & 0.0 & & \\
\hline
\end{tabular}




\subsection{Safety}

We found no difference between CNI- and Belatacept-treated patients concerning all safety endpoints, except for severe infection (Figure 1, Table 5).

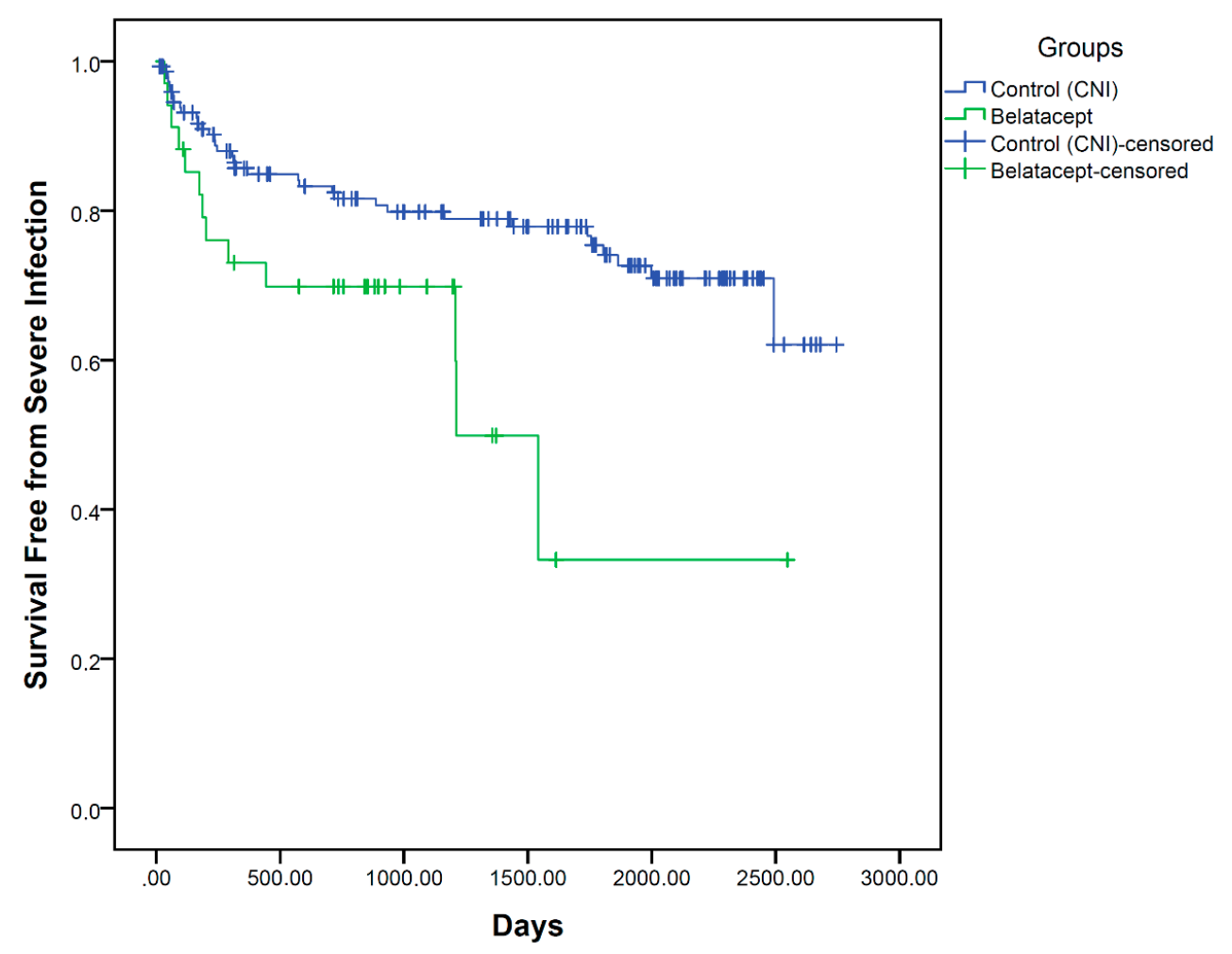

Figure 1. KM-Plot for severe infection ( $\log -\operatorname{Rank} p=0.013)$, defined as infection leading to the admission of the patient to hospital.

Concerning severe infections, defined as infections leading to the admission of the patient to hospital, we found more infections in the Belatacept group ( 38.2 vs. $23.3 \%$, Log-Rank $p=0.013$ ). The type of severe infection also differed between the groups, with a higher proportion of diarrhoea, urinary tract infections and sepsis, but fewer instances of pneumonia, in Belatacept-treated patients. In the univariate Cox regression analysis, Belatacept treatment, number of postoperative HDs, the presence of creatinine at discharge, ECD and donor age were significant risk factors for severe infections (Table 6), while the male sex of the recipient was a protective factor. In the multivariate analysis, no risk factor remained significant (including Belatacept), whereas the male sex of the recipient remained a significant protective factor for severe infection in our cohort. It is noteworthy that Belatacept was not a significant risk factor in any Cox regression analysis for all other safety endpoints. Risk factors for CMV reactivation were number of postoperative HDs (HR 1.123, 95\%CI 1.050-1.201, $p=0.001$ ) and the presence of serum-creatinine at discharge (HR 1.335, 95\%CI 1.087-1.638, $p=0.006$ ) of which none remained significant in a multivariate model. A risk factor for BKV reactivation in patients' plasma was treatment with steroids at follow-up (HR 3.358, 95\%CI 1.246-9.051, $p=0.017$ ) whereas the male sex of the donor was protective (HR $0.362,95 \% \mathrm{CI} 0.142-0.917, p=0.032$ ). The treatment with steroids at follow-up remained significant in the multivariate model (HR 2.850, 95\%CI 1.042-7.796, $p=0.041$ ). BKV reactivation in patients' urine was significantly correlated with recipient (HR 1.041, 95\%CI 1.013-1.069, $p=0.004$ ) and donor age (HR 1.020, 95\%CI 1.001-1.038, $p=0.036$ ), of which recipient age remained multivariately significant (HR 1.033, 95\%CI 1.003-1.064, $p=0.029$ ). 
Table 6. Cox regression for severe infection. All the significant risk factors from the univariate Cox-Regression are shown and were included in the multivariate analysis. $p$-values $<0.05$ are marked with an asterisk *.

\begin{tabular}{ccccccc}
\hline \multirow{2}{*}{ Factor } & \multicolumn{3}{c}{ Univariate Analysis } & \multicolumn{3}{c}{ Multivariate Analysis ${ }^{1}$} \\
\cline { 2 - 7 } & HR & $\mathbf{9 5 \% - C I}$ & $p$ & HR & 95\%-CI & $p$ \\
\hline Belatacept & 2.236 & $1.163-4.301$ & $0.016^{*}$ & 1.363 & $0.659-2.819$ & 0.403 \\
Male recipient Sex & 0.548 & $0.305-0.983$ & $0.044^{*}$ & 0.459 & $0.242-0.870$ & $0.017^{*}$ \\
Number of PO HDs & 1.156 & $1.062-1.259$ & $0.001^{*}$ & 1.057 & $0.939-1.189$ & 0.358 \\
Creatinine at & 1.602 & $1.255-2.044$ & $<0.001^{*}$ & 1.131 & $0.628-2.037$ & 0.682 \\
discharge & 2.867 & $1.578-5.209$ & $0.001^{*}$ & 1.373 & $0.488-3.858$ & 0.548 \\
$\quad$ ECD & 1.032 & $1.012-1.052$ & $0.002^{*}$ & 1.019 & $0.985-1.054$ & 0.287 \\
Donor Age & &
\end{tabular}

${ }_{1}^{1}$ adjusted for belatacept treatment, male recipient sex, number of post-operative (PO) hemodialysis sessions (HDs), creatinine ad discharge, extended criteria donor (ECD), donor age.

The only univariate risk factor for the safety endpoint de novo cardiovascular events was pre-existing cerebrovascular disease (HR 6.144, 95\%CI 1.026-36.798, $p=0.047$ ). All other parameters, and especially Belatacept (HR 1.938, 95\%CI 0.214-17.591, $p=0.557$ ), were not significant. Concerning malignancy, we found no significant factor in the univariate Cox regression. Univariate risk factors for death were recipient age (HR 1.044, 95\%CI 1.006-1.083, $p=0.023$ ), HLA mismatch (HR 2.27, 95\%CI $1.011-5.099, p=0.047$ ) and the presence of creatinine at discharge (HR 1.350, 95\%CI 1.018-1.791, $p=0.037)$. Recipient age was the only significant risk factor for death in multivariate Cox regression (HR 1.036, 95\%CI 1.001-1.074, $p=0.046$ ).

\section{Discussion}

We conducted a retrospective cohort study of renal allograft recipients at high cardiovascular risk either treated with a Belatacept- or CNI-based immunosuppressive regimen. eGFR improved with Belatacept treatment, but slightly decreased during CNI therapy and, in the multivariate analysis, Belatacept treatment was the only significant factor for the improvement of $\triangle \mathrm{eGFR}$. This is in line with the BENEFIT and BENEFIT-EXT studies, which also demonstrated an increase of GFR over a follow-up of seven years [12-14]. However, the CNI comparator cohort consisted of cyclosporine A-treated patients only, whereas our group was mainly treated with tacrolimus (68\% of patients). Our recipients were of a similar age (56 years) to recipients in BENEFIT-EXT, but older compared to BENEFIT (43 years). Additionally, our CV high risk cohorts consisted of more diabetic patients (CNI group: $57 \%$, Belatacept group: $32 \%$ vs. BENEFIT: $15 \%$, BENEFIT-EXT: $16 \%$ ) and had worse donor characteristics (living donors: CNI group $4 \%$, Belatacept group $15 \%$ vs. BENEFIT 58\%, BENEFIT-EXT not reported). Furthermore, the BENEFIT studies did not report the number of patients suffering from established cardiovascular disease, which was substantial in our Belatacept $(94 \%)$ and CNI patients (75\%). Nevertheless, and although Belatacept patients had inferior renal allograft function at the time of discharge after transplantation, serum creatinine levels and eGFR were similar at follow-up in this high CV risk cohort compared to CNI-treated patients. Bertrand et al. [15] and Le Meur et al. [16] reported similar results in 17 and 25 patients treated with Belatacept, because of vascular damage and CNI intolerance.

Belatacept was not associated with an increased risk of rejection in our patients. BENEFIT-EXT [17] reported a higher risk in Belatacept-treated patients, whereas BENEFIT [18] found no significant difference. However, our CNI cohort was mainly treated with tacrolimus, which is generally accepted to have a slightly higher immunosuppressive potential, rather than cyclosporine A as in the BENEFIT(-EXT) studies. Belatacept was not a risk factor for graft loss in our cohort (HR 0.987, 95\%CI 0.283-3.417, $p=0.980$ ), which is in line with the literature $[17,18]$.

Concerning safety and in contrast to Florman et al. [13], we found that Belatacept treatment was associated with an increased incidence of severe infections in the univariate Cox regression. The most 
obvious differences were higher event rates of diarrhoea, urinary tract infection and sepsis. Sepsis as an endpoint has not been reported in any previous studies of Belatacept patients. In line with our data, gastroenteritis and urinary tract infections were numerically higher in the switch studies $[7,8,19]$. However, in the multivariate regression analysis, Belatacept treatment was not associated with an increase of this endpoint. One explanation for the higher incidence of gastroenteritis (diarrhoea) might be the more frequent use of mycophenlate in Belatacept patients (76.47 vs. $60.67 \%$; 1.26 -fold more), which is known to have such gastrointestinal side-effects. The reason for this is that physicians tend to prescribe the triple combination of mycophenolic acid, steroids and Belatacept, as only this regimen was approved for renal allograft recipients. However, it cannot be ruled out that some examples of mycophenolate-associated diarrhoea have been misdiagnosed as infectious diarrhoea, therefore increasing the proportion of diarrhoea in Belatacept patients compared to CNI patients, although the fold-change of diarrhoea ( $8.8 \mathrm{vs.} 0.7 \%, 12.57$-fold) substantially exceeds the use of mycophenlate in the Belatacept compared to the CNI group (1.26-fold, see above). Additionally, the proportion of mycophenolate-treated patients in the switch studies was almost identical between the groups (and higher compared to our data (approx. 94\%)) [7,8,19].

In our cohort, Belatacept treatment was not a risk factor for CMV reactivation, malignancy or death. This is in line with the published data cited above. Additionally, Belatacept treatment was not a risk factor for BKV reactivations either in patients' serum or urine. Unfortunately, data on these endpoints were not reported in the BENEFIT and BENEFIT-EXT studies. Nevertheless, our data is in line with the phase II studies, which showed only slightly increased cumulative incidence rates $(0.85$ vs. 0 [19]) and events (4 vs. $\%$ [8] and 2 vs. 0\% [7]) in Belatacept patients. Unfortunately, no statistics were calculated in these studies.

Published data suggest a beneficial impact of Belatacept on arterial stiffness and metabolic parameters (e.g., arterial hypertension and lipid profile) or post-transplant diabetes mellitus. The authors concluded that this could improve kidney transplant recipients' survival by reducing events related to those factors $[9,17,18,20]$. However, available data from the long-term outcomes of these studies do not show a significant difference in severely adverse events (including cardiac or vascular disorders) [12,14]. Concerning patients with high cardiovascular risk, the only study that has been published so far was a post-hoc analysis of patients with pre-existing diabetes of the BENEFIT and BENEFIT-EXT cohorts. Patient survival and renal function were numerically but not significantly higher in Belatacept patients at 12 months' follow-up and, unfortunately, cardiovascular events were not reported [8]. Hence, to the best of our knowledge, this is the first report on cardiovascular events in Belatacept compared to CNI-treated patients. We found no difference between Belatacept- and CNI-treated patients concerning de novo cardiovascular events with a cumulative follow-up of 1194 months in Belatacept $(n=35)$ and 7309 months in CNI patients $(n=150)$.

Our study has limitations. Firstly, this is a retrospective study, which by nature does not provide the same data quality as a prospective design. Secondly, the size of the study population is relatively small, as we included only 34 Belatacept patients and $150 \mathrm{CNI}$ patients as a comparator. Thirdly, the baseline characteristics of time on RRT, primary renal disease, diabetes, cardiovascular disease and arterial hypertension were different between our two populations (Table 1) and it is possible that statistical methods were not able to correct appropriately for this issue. Fourthly, the median time of follow-up was longer in CNI patients (Belatacept: 1054 vs. CNI: 1462 days) but not statistically significant $(p=0.084)$. From our point of view, the duration of follow-up is still significant, although one might argue that a longer follow-up would have been beneficial, especially for the end point "cardiovascular event". However, the number of studies that have published data of renal allograft recipients on Belatacept-based immunosuppression is generally limited. In total, until the end of 2014, the data of 521 Belatacept patients, which were compared to CNI-treated controls, were published [21]. Recently, one study of 17 Belatacept patients matched to 18 control patients, and two studies of 25 and 6 cases that were converted from CNI to Belatacept without a control population, were published $[15,16,22]$. The randomized controlled trials BENEFIT [18], BENEFIT-EXT [17] and the switch study [8] originally 
reported one-year results of 181, 129 and 81 belatacept patients compared to a CNI-treated cohort of similar size. Hence, we believe that our cohort size and follow-up period is considerable and contributes information in a real world setting.

In conclusion, we believe that Belatacept is an efficient, beneficial and safe option for renal allograft recipients at high cardiovascular risk. In our cohort, Belatacept treatment was associated with a superior graft function compared to a CNI-treated cohort and was not a risk factor for renal allograft rejection, -loss, severe infection, CMV- or BKV-reactivation, malignancy or death.

Author Contributions: Conceptualization: H.N.; Data curation: H.N., I.L.-L., M.E., F.P., N.P., J.M., H.S.-M., M.R., P.S.; Formal analysis: H.N., M.E., F.P., N.P., J.M., M.R.; Methodology: H.N., J.K., G.M.; Project administration: H.N., G.M.; Software: H.N.; Supervision: H.N.; Validation: H.N., I.L.-L., M.R.; Writing-original draft: H.N., M.R., P.S., I.E.E., G.M.; Writing-review \& editing: H.N., I.E.E., G.M.

Funding: This research received no external funding.

Conflicts of Interest: The authors declare no conflict of interest.

\section{Abbreviations}

$\begin{array}{ll}\text { BKV } & \text { polyoma virus } \\ \text { BMI } & \text { body mass index } \\ \text { CAD } & \text { coronary artery disease } \\ \text { CD } & \text { cluster of differentiation } \\ \text { CMV } & \text { cytomegalo virus } \\ \text { CNI } & \text { calcineurin inhibitor } \\ \text { CV } & \text { cardiovascular } \\ \text { CVA } & \text { cerebrovascular event } \\ \text { DD } & \text { deceased donor } \\ \text { ECD } & \text { extended criteria donor } \\ \text { eGFR } & \text { estimated glomerular filtration rate } \\ \text { HD } & \text { hemodialysis } \\ \text { HLA } & \text { human leukocyte antigen } \\ \text { HR } & \text { hazard ratio } \\ \text { IQR } & \text { interquartile range } \\ \text { mTORi } & \text { mammalian target of rapamycin inhibitor } \\ \text { PAD } & \text { peripheral artery disease } \\ \text { PCR } & \text { polymerase chain reaction } \\ \text { PO } & \text { post-operative after renal transplantation } \\ \text { RRT } & \text { renal replacement therapy } \\ \text { RTx } & \text { renal transplantation } \\ \text { SAB } & \text { subarachnoideal bleeding } \\ \text { SDH } & \text { subdural hematoma } \\ \text { 95\%CI } & \text { 95\% confidence interval } \\ & \end{array}$

\section{References}

1. Lamb, K.E.; Lodhi, S.; Meier-Kriesche, H.U. Long-term renal allograft survival in the United States: A critical reappraisal. Am. J. Transplant. 2011, 11, 450-462. [CrossRef] [PubMed]

2. Stallone, G.; Infante, B.; Grandaliano, G.; Gesualdo, L. Management of side effects of sirolimus therapy. Transplantation 2009, 87 (Suppl. 8), S23-S26. [CrossRef]

3. Ekberg, H.; Tedesco-Silva, H.; Demirbas, A.; Vítko, S.; Nashan, B.; Gürkan, A.; Margreiter, R.; Hugo, C.; Grinyó, J.M.; Frei, U.; et al. Reduced exposure to calcineurin inhibitors in renal transplantation. N. Engl. J. Med. 2007, 357, 2562-2575. [CrossRef] [PubMed]

4. Latek, R.; Fleener, C.; Lamian, V.; Kulbokas, E., 3rd; Davis, P.M.; Suchard, S.J.; Curran, M.; Vincenti, F.; Townsend, R. Assessment of belatacept-mediated costimulation blockade through evaluation of CD80/86-receptor saturation. Transplantation 2009, 87, 926-933. [CrossRef] [PubMed] 
5. Vincenti, F.; Larsen, C.P.; Alberu, J.; Bresnahan, B.; Garcia, V.D.; Kothari, J.; Lang, P.; Urrea, E.M.; Massari, P.; Mondragon-Ramirez, G.; et al. Three-year outcomes from BENEFIT: A randomized: Active-controlled: Parallel-group study in adult kidney transplant recipients. Am. J. Transplant. 2012, 12, 210-217. [CrossRef] [PubMed]

6. Vincenti, F.; Rostaing, L.; Grinyo, J.; Rice, K.; Steinberg, S.; Gaite, L.; Moal, M.C.; Mondragon-Ramirez, G.A.; Kothari, J.; Polinsky, M.S.; et al. Belatacept and long-term outcomes in kidney transplantation. N. Engl. J. Med. 2016, 374, 333-343. [CrossRef] [PubMed]

7. Grinyo, J.; Alberu, J.; Contieri, F.L.; Manfro, R.C.; Mondragon, G.; Nainan, G.; Rial Mdel, C.; Steinberg, S.; Vincenti, F.; Dong, Y.; et al. Improvement in renal function in kidney transplant recipients switched from cyclosporine or tacrolimus to belatacept: 2-year results from the long-term extension of a phase II study. Transpl. Int. 2012, 25, 1059-1064. [CrossRef] [PubMed]

8. Rostaing, L.; Massari, P.; Garcia, V.D.; Mancilla-Urrea, E.; Nainan, G.; del Carmen Rial, M.; Steinberg, S.; Vincenti, F.; Shi, R.; Di Russo, G.; et al. Switching from calcineurin inhibitor-based regimens to a belatacept-based regimen in renal transplant recipients: A randomized phase II study. Clin. J. Am. Soc. Nephrol. 2011, 6, 430-439. [CrossRef]

9. Melilli, E.; Manonelles, A.; Montero, N.; Grinyo, J.; Martinez-Castelao, A.; Bestard, O.; Cruzado, J. Impact of immunosuppressive therapy on arterial stiffness in kidney transplantation: Are all treatments the same? Clin. Kidney J. 2018, 11, 413-421. [CrossRef]

10. Melilli, E.; Bestard-Matamoros, O.; Manonelles-Montero, A.; Sala-Bassa, N.; Mast, R.; Grinyo-Boira, J.M.; Cruzado, J.M. Arterial stiffness in kidney transplantation: A single center case-control study comparing belatacept versus calcineurin inhibitor immunosuppressive based regimen. Nefrologia 2015, 35, 58-65. [CrossRef]

11. Seibert, F.S.; Steltzer, J.; Melilli, E.; Grannas, G.; Pagonas, N.; Bauer, F.; Zidek, W.; Grinyo, J.; Westhoff, T.H. Differential impact of belatacept and cyclosporine A on central aortic blood pressure and arterial stiffness after renal transplantation. Clin. Transplant. 2014, 28, 1004-1009. [CrossRef] [PubMed]

12. Durrbach, A.; Pestana, J.M.; Florman, S.; Del Carmen Rial, M.; Rostaing, L.; Kuypers, D.; Matas, A.; Wekerle, T.; Polinsky, M.; Meier-Kriesche, H.U.; et al. Long-term outcomes in belatacept- versus cyclosporine-treated recipients of extended criteria donor kidneys: Final results from benefit-ext: A phase III randomized study. Am. J. Transplant. 2016, 16, 3192-3201. [CrossRef] [PubMed]

13. Florman, S.; Becker, T.; Bresnahan, B.; Chevaile-Ramos, A.; Carvalho, D.; Grannas, G.; Muehlbacher, F.; O'Connell, P.J.; Meier-Kriesche, H.U.; Larsen, C.P. Efficacy and safety outcomes of extended criteria donor kidneys by subtype: Subgroup analysis of BENEFIT-EXT at 7 years after transplant. Am. J. Transplant. 2017, 17, 180-190. [CrossRef] [PubMed]

14. Rostaing, L.; Vincenti, F.; Grinyo, J.; Rice, K.M.; Bresnahan, B.; Steinberg, S.; Gang, S.; Gaite, L.E.; Moal, M.C.; Mondragon-Ramirez, G.A.; et al. Long-term belatacept exposure maintains efficacy and safety at 5 years: Results from the long-term extension of the BENEFIT study. Am. J. Transplant. 2013, 13, 2875-2883. [CrossRef] [PubMed]

15. Bertrand, D.; Cheddani, L.; Etienne, I.; Francois, A.; Hanoy, M.; Laurent, C.; Lebourg, L.; Le Roy, F.; Lelandais, L.; Loron, M.C.; et al. Belatacept rescue therapy in kidney transplant recipients with vascular lesions: A case control study. Am. J. Transplant. 2017, 17, 2937-2944. [CrossRef] [PubMed]

16. Le Meur, Y.; Aulagnon, F.; Bertrand, D.; Heng, A.E.; Lavaud, S.; Caillard, S.; Longuet, H.; Sberro-Soussan, R.; Doucet, L.; Grall, A.; et al. Effect of an early switch to belatacept among calcineurin inhibitor-intolerant graft recipients of kidneys from extended-criteria donors. Am. J. Transplant. 2016, 16, 2181-2186. [CrossRef]

17. Durrbach, A.; Pestana, J.M.; Pearson, T.; Vincenti, F.; Garcia, V.D.; Campistol, J.; Rial Mdel, C.; Florman, S.; Block, A.; Di Russo, G.; et al. A phase III study of belatacept versus cyclosporine in kidney transplants from extended criteria donors (BENEFIT-EXT study). Am. J. Transplant. 2010, 10, 547-557. [CrossRef] [PubMed]

18. Vincenti, F.; Charpentier, B.; Vanrenterghem, Y.; Rostaing, L.; Bresnahan, B.; Darji, P.; Massari, P.; Mondragon-Ramirez, G.A.; Agarwal, M.; Di Russo, G.; et al. A phase III study of belatacept-based immunosuppression regimens versus cyclosporine in renal transplant recipients (BENEFIT study). Am. J. Transplant. 2010, 10, 535-546. [CrossRef] 
19. Grinyo, J.M.; Del Carmen Rial, M.; Alberu, J.; Steinberg, S.M.; Manfro, R.C.; Nainan, G.; Vincenti, F.; Jones-Burton, C.; Kamar, N. Safety and efficacy outcomes 3 years after switching to belatacept from a calcineurin inhibitor in kidney transplant recipients: Results from a phase 2 randomized trial. Am. J. Kidney Dis. 2017, 69, 587-594. [CrossRef]

20. Wissing, K.M.; Pipeleers, L. Obesity: Metabolic syndrome and diabetes mellitus after renal transplantation: Prevention and treatment. Transplant. Rev. (Orlando) 2014, 28, 37-46. [CrossRef]

21. Masson, P.; Henderson, L.; Chapman, J.R.; Craig, J.C.; Webster, A.C. Belatacept for kidney transplant recipients. Cochrane Database Syst Rev 2014. [CrossRef] [PubMed]

22. Gupta, G.; Regmi, A.; Kumar, D.; Posner, S.; Posner, M.P.; Sharma, A.; Cotterell, A.; Bhati, C.S.; Kimball, P.; Massey, H.D.; et al. Safe conversion from tacrolimus to belatacept in high immunologic risk kidney transplant recipients with allograft dysfunction. Am. J. Transplant. 2015, 15, 2726-2731. [CrossRef] [PubMed]

(C) 2019 by the authors. Licensee MDPI, Basel, Switzerland. This article is an open access article distributed under the terms and conditions of the Creative Commons Attribution (CC BY) license (http://creativecommons.org/licenses/by/4.0/). 